\title{
Expression of the neural cell adhesion molecule and polysialic acid in human neuroblastoma cell lines
}

\author{
URSULA VALENTINER $^{1}$, MARTINA MÜHLENHOFF ${ }^{2}$, ULRICH LEHMANN ${ }^{3}$, \\ HERBERT HILDEBRANDT $^{2}$ and UDO SCHUMACHER ${ }^{1}$ \\ ${ }^{1}$ Institute of Anatomy II: Experimental Morphology, University Hospital Hamburg-Eppendorf, \\ Martinistr. 52, 20246 Hamburg; ${ }^{2}$ Institute of Cellular Chemistry and ${ }^{3}$ Institute of Pathology, \\ Medical School Hannover, Carl-Neuberg-Str. 1, 30625 Hannover, Germany
}

Received February 25, 2011; Accepted April 6, 2011

DOI: $10.3892 /$ ijo. 2011.1038

\begin{abstract}
The neural cell adhesion molecule NCAM is a cell surface glycoprotein of the immunoglobulin superfamily and is widely expressed in tumours of neuroectodermal origin such as neuroblastoma. NCAM can be decorated by the carbohydrate polymer polysialic acid (polySia), which attenuates NCAM-mediated cell adhesion and increases cellular motility. The key enzymes in the biosynthesis of polySia are the two polysialyltransferases ST8SiaII and ST8SiaIV. In the present study, expression of NCAM, polySiaNCAM, ST8SiaII and ST8SiaIV was investigated in five human neuroblastoma cell lines before and after xenografting into SCID mice by immunohistochemistry, Western blot analysis and real-time PCR. Results were correlated with the metastatic potential. In vitro, three cell lines (LAN-1, LAN-5 and SH-SY5Y) were positive for polySia attached to the transmembrane isoforms NCAM-140 and NCAM-180, whereas Kelly and SK-N-SH cells were negative for NCAM and polySia. In the presence of NCAM, the level of polySia correlated with the amount of polysialyltransferase transcripts, which were highest in LAN-1, LAN-5 and SH-SY5Y cells. In the respective primary tumours grown in SCID mice, the expression patterns of NCAM, polySia and polysialyltransferases were similar to those observed in vitro. After subcutaneous engraftment, polySia-NCAM-positive neuroblastoma developed disseminated micrometastases, a metastatic pattern that was not observed for tumours derived from NCAM-negative cell lines. Together, this indicates that the presence of polySia reduces the adhesiveness of tumour cells and promotes dissemination.
\end{abstract}

Correspondence to: Dr Ursula Valentiner, Institute of Anatomy II: Experimental Morphology, University Hospital Hamburg-Eppendorf, Martinistr. 52, 20246 Hamburg, Germany

E-mail: valentin@uke.uni-hamburg.de

Key words: neural cell adhesion molecule, polysialic acid, neuroblastoma, SCID mouse, metastasis

\section{Introduction}

Neuroblastoma is the most common extracranial solid tumour in children. It is derived from embryonic neural crest cells of the peripheral sympathetic nervous system and can occur anywhere along the strands of the sympathetic nervous system (1). The tumour is noted for its variable clinical and biological behaviour, but most children over the age of one year present with high-risk disease with long-term survival still $<40 \%$ $(1,2)$. Therefore reliable markers with prognostic potential for the different clinical stages and the search for new therapeutic concepts are of great clinical importance.

Like other malignant tumours of neuroectodermal origin, nearly all neuroblastomas express the neural cell adhesion molecule (NCAM) $(3,4)$. NCAM is a cell surface glycoprotein of the immunoglobulin (Ig) superfamily and by extensive alternative mRNA splicing a variety of NCAM isoforms can be generated from a single-copy gene (5). All isoforms can be grouped into three classes: NCAM-120, a glycosylphosphatidylinositol (GPI) anchored form predominantly found in normal and differentiated tissue (6), as well as NCAM-140 and NCAM-180 which are both transmembrane proteins that differ in the length of their cytoplasmic domain. These two isoforms are mostly expressed in less differentiated or malignant cell types $(4,6,7)$.

NCAM mediates cell-cell adhesion by a calciumindependent homophilic interaction between NCAM molecules on opposite cells (8); on the other hand it can act as a plasticitypromoting molecule by decreasing the overall cell-adhesion (9). The key regulator between these two opposite functions of NCAM is polysialic acid (polySia), a dynamically regulated posttranslational modification found in mammals exclusively on NCAM. Polysialic acid is a unique linear homopolymer with up to 90 sialic acid residues in $\alpha 2,8$-glycosidic linkage (10). The presence of this large polyanionic carbohydrate moiety attenuates NCAM-mediated cell adhesion and increases cellular motility (10).

Polysialic acid represents an oncodevelopmental antigen that is re-expressed on several malignant neuroectodermal and neuroendocrine tumours, including neuroblastoma (10). Several studies indicate that downregulation of NCAM or polysialylation of NCAM reduces the adhesiveness of tumour 
cells and facilitates the detachment of cells during the first steps of the metastatic cascade $(11,12)$. Thus, expression of polysialic acid has been shown to correlate with tumour progression, metastasis and invasion in several tumours $(13,14)$. In neuroblastoma expression of polySia-NCAM was highest in patients with advanced stage of disease and correlated with other prognostic markers, such as MYCN amplification (15). Furthermore serum levels of polySia-NCAM were shown to correlate with tumour content and were found to decrease during successful therapy (16).

The key enzymes in the biosynthesis of polySia are the two closely related polysialyltransferases ST8SiaII and ST8SiaIV, formerly named STX and PST, respectively (17). ST8SiaII is described as the predominant form in embryonic and early postnatal brain, whereas ST8SiaIV was found to be the major form in adult brain (18-20). Expression of ST8SiaII mRNA was recently shown to be a potential molecular marker of metastatic neuroblastoma (21).

Human tumour cell xenograft models using immunodeficient mice have provided an in vivo model system for the study of prognostic markers (22). Based on our previous study demonstrating that the SCID mouse model is ideally suited to study metastasis of human neuroblastoma cell lines (23), we now used this xenograft model to investigate the role of polySia-NCAM and the two polysialyltransferases for tumour growth and metastasis. Therefore, the expression level of ST8SiaII and ST8SiaIV as well as polySia-NCAM and the NCAM isoform pattern were investigated in five human neuroblastoma cell lines before and after xenografting into SCID mice and results were correlated with the metastatic potential.

\section{Materials and methods}

Cell lines. Five human neuroblastoma cell lines, namely Kelly, LAN-1, LAN-5, SH-SY5Y and SK-N-SH were investigated in this study. Kelly, LAN-1, LAN-5 and SK-N-SH were a kind gift from Dr R. Erttmann (Department of Paediatric Haematology and Oncology, University Medical Center Hamburg-Eppendorf, Germany); SH-SY5Y cells were obtained from Dr H. Hildebrandt (Institute of Cellular Chemistry, Medical School Hannover, Germany). The cell lines were cultured in vitro under standard cell culture conditions $\left(37^{\circ} \mathrm{C}, 100 \%\right.$ relative humidity, $\left.5 \% \mathrm{CO}_{2}\right)$ in RPMI medium (Gibco/Life Technologies, Karlsruhe, Germany) supplemented with $10 \%$ heat-inactivated fetal bovine serum (FBS, Gibco), 2 mM L-glutamine (Gibco), 100 U/ml penicillin and $100 \mu \mathrm{g} / \mathrm{ml}$ streptomycin (Gibco). Before reaching confluence, cells were routinely harvested for passaging using $0.05 \%$ trypsin- $0.02 \%$ EDTA (Gibco). Prior to all experiments, all cells were checked for the absence of mycoplasma infections using the VenorGeM ${ }^{\circledR}$-Mycoplasma detection kit (Minerva Biolabs GmbH, Berlin, Germany).

For the production of cytospins, cells were harvested using $0.05 \%$ trypsin- $0.02 \%$ EDTA (Gibco). A $200 \mu$ l cell suspension $\left(\sim 10^{6}\right.$ cells $\left./ \mathrm{ml}\right)$ was centrifuged onto HistoBond ${ }^{\circledR}$ microscope slides (Marienfeld, Lauda-Königshofen, Germany) at 1500 x g and $4 \mathrm{~min}$ at $20^{\circ} \mathrm{C}$ using $1-\mathrm{cm}$ diameter cytocentrifuge funnel and Hettich centrifuge (Hettich, Tuttlingen, Germany). The supernatant was withdrawn and the cells were air dried overnight. Cytospins were fixed in acetone for $15 \mathrm{~min}$ at room temperature before immunohistochemistry.
For injection into pathogen-free BALB/c severe combined immunodeficient (SCID) SCID/SCID mice, neuroblastoma cells were harvested by trypsination and viable cells $\left(5 \times 10^{6}\right)$ were suspended in $1 \mathrm{ml}$ cell culture medium. An aliquot of $200 \mu \mathrm{l}$ of this suspension was injected subcutaneously between the scapulae of each SCID mouse. The mice bearing neuroblastomas were sacrificed when the tumour had reached maximal growth (up to $10 \%$ of the body weight of the animal at the beginning of the experiment) or started to ulcerate. Primary tumours were removed and divided into several parts. One part of the tumour was frozen for cryosectioning. The animal experiment was approved by the local animal experiment approval committee (Behörde für Soziales, Familie, Gesundheit, Verbraucherschutz; Amt für Gesundheit und Verbraucherschutz, Hamburg, Germany, project no. 56/03) and was performed in compliance with local animal welfare laws, guidelines and policies.

Antibodies and enzymes. NCAM-specific mouse monoclonal antibody (mAb) 123C3 (24) and polySia-specific mAb 735 (25) were used after affinity purification on Protein G- and Protein A-sepharose (GE Healthcare), respectively. Endosialidase (endoN) of bacteriophage K1F was purified as described previously (26).

Immunohistochemistry. Immunoreaction of the neuroblastoma cells was examined on cytospins of in vitro grown cells and cryosections of neuroblastomas grown in SCID mice. The following monoclonal antibodies were used: polySia-specific mouse mAb 735 (anti-polySia) and NCAM-specific mouse $\mathrm{mAb} 123 \mathrm{C} 3[5 \mu \mathrm{g} / \mathrm{ml}$ in $3 \%$ BSA (bovine serum albumin) in PBS, respectively]. Briefly, sections incubated with mAb 735 and $\mathrm{mAb} 123 \mathrm{C} 3$ were treated twice with lysine $(18.3 \mathrm{mg} / \mathrm{ml})$ in PBS (phosphate-buffered saline, $\mathrm{pH}$ 7.6) for $15 \mathrm{~min}$ and rinsed in PBS. Non-specific binding was blocked by incubating the sections with $3 \%$ BSA (bovine serum albumin) and $0.3 \%$ Triton X-100 in PBS for $30 \mathrm{~min}$ at room temperature. Sections were incubated overnight at $4^{\circ} \mathrm{C}$ with primary antibody, rinsed, and incubated for $30 \mathrm{~min}$ at room temperature with a respective biotinylated secondary antibody (Dako) diluted 1:200 in PBS and 3\% BSA. Subsequently, sections were rinsed and incubated for $30 \mathrm{~min}$ with a streptavidin-alkaline phosphatase kit (Vector Laboratories). Enzyme reactivity of the alkaline phosphatase complex was visualized using NaphtolAS-biphosphate as a substrate and hexatozised New Fuchsin for simultaneous coupling. Sections were counterstained with Mayer's hemalum diluted 1:1 in distilled water for 5-10 sec, rinsed in tap water and covered with Crystal Mount and Clarion (Biomeda, Foster City, CA). They were examined and photographed under a Zeiss Axioplan photomicroscope.

Specifity of mAb 735 was controlled on samples pretreated with $1 \mu \mathrm{g} / \mathrm{ml}$ endosialidase in $3 \%$ BSA and $0.3 \%$ Triton $\mathrm{X}-100$ in PBS for $2 \mathrm{~h}$ at $37^{\circ} \mathrm{C}$, an enzyme that specifically degrades polySia. These controls showed an insignificant background staining. Negative controls were treated the same way, omitting the incubation step with the primary antibody, and showed no immunoreactivity.

Protein extraction and Western blot analysis. Neuroblastoma cells $\left(5 \times 10^{6}\right)$ were washed twice with phosphate-buffered 


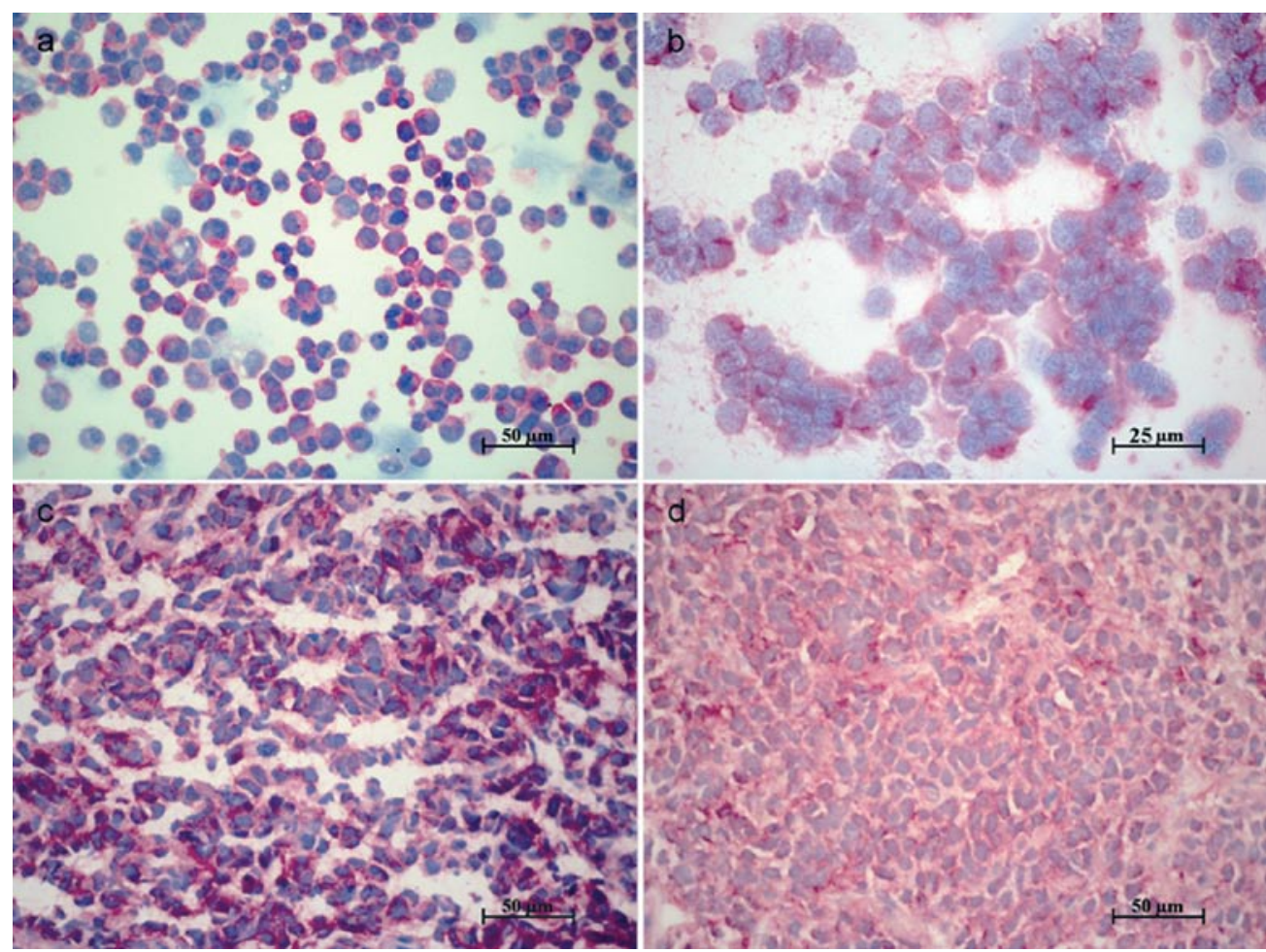

Figure 1. NCAM (a and c) and polySia (b and d) immunohistochemistry of SH-SY5Y neuroblastoma cells grown in vitro and LAN-5 cells grown in SCID mice. SH-SY5Y cells showed a moderate immunostaining with anti-NCAM antibody mAb 123C3 (a) and anti-polySia antibody mAb 735 (b). LAN-5 primary tumours showed a moderate to strong NCAM expression (c) and a moderate polySia expression (d).

saline and lysed in $300 \mu \mathrm{l}$ of ice-cold lysis buffer $(20 \mathrm{mM}$ Tris- $\mathrm{HCl}$ pH 8.0, $150 \mathrm{mM} \mathrm{NaCl}, 5 \mathrm{mM}$ EDTA, $1 \mathrm{mM}$ PMSF, $70 \mu \mathrm{g} / \mathrm{ml}$ aprotinin, $10 \mu \mathrm{g} / \mathrm{ml}$ leupeptin, $2 \%$ Triton $\mathrm{X}-100)$. Detergent extracts were clarified by centrifugation and one aliquot was treated with $20 \mathrm{ng} / \mu \mathrm{l}$ endoN for $45 \mathrm{~min}$ on ice. For xenograft tumours $200 \mu \mathrm{l}$ of lysis buffer was used per $100 \mathrm{mg}$ wet weight and homogenization was performed in a Precellys 24 homogenizer using 1.4-mm beads (Peqlab). Proteins were separated by SDS-PAGE (30 $\mu \mathrm{g}$ total protein per lane) and electroblotted on nitrocellulose membranes. Equal loading and protein transfer were controlled by Ponceau S staining. After removal of the dye, immunostaining was carried out using anti-polySia mAb 735 or anti-NCAM mAb $123 \mathrm{C} 3$ in a concentration of $2.5 \mu \mathrm{g} / \mathrm{ml}$. Bound antibodies were detected with peroxidase-coupled anti-mouse $\mathrm{IgG}$ and developed by enhanced chemiluminescence.

RNA isolation, cDNA synthesis and quantitative real-time $P C R$. Total RNA was extracted from cells using TRIzol reagent (Invitrogen, Karlsruhe, Germany) and treated with RQ1 DNase I (Promega, Mannheim, Germany), according to the manufacturer's recommendations. The cDNA synthesis was performed using Superscript II reverse transcriptase (Invitrogen) and $1 \mu \mathrm{g}$ of DNAse I-treated total RNA as template in a $20 \mu \mathrm{l}$ reaction volume.

For quantitative gene expression analyses $1 \mu \mathrm{l}$ cDNA was subjected to PCR amplification using SYBR-Green (Invitrogen) and the ABI Prism 7700 system (Applied Biosystems, Darmstadt, Germany). Each PCR reaction of $25 \mu$ l contained $2.5 \mu 110 \mathrm{X}$ concentrated universal PCR buffer (Invitrogen), 1.5 or $2.5 \mu \mathrm{l} \mathrm{MgCl}_{2}(25 \mathrm{mM}), 0.5 \mu \mathrm{ldNTP}$ 's (10 mM), $0.25 \mu \mathrm{l}$
ROX reference dye (Invitrogen), $2 \mu \mathrm{l} \mathrm{SYBR-Green(Invitrogen),}$ $0.6 \mu \mathrm{lmixed}$ forward and reverse primer $10 \mathrm{pmol} / 1 \mathrm{each}$, $0.125 \mu$ l Platinum Taq DNA polymerase (5 U/ $\mu$ l, Invitrogen) and $1 \mu \mathrm{l}$ cDNA. The following primer pairs were used: STIIf 5'-TGACGCCCACAGCTTCG-3') and STIIr (5'-CATCCCG GGCATACTCCTG-3') for ST8SIA2, STIVf (5'GACAA AAGAAATAGCAAGAACTGAGGA-3') and ST-IVr (5'-CCGACTCAAAGACAATTCACCA-3') for ST8SIA4, hNCAMf (5'-GAGATCAGCGTTGGAGAGTCC-3') hNCAMr (5'-GGAGAACCAGGAGATGTCTTTATCTT-3') for NCAM1, $\beta G U S f$ (5'-CTCATTTGGAATTTTGCCGATT-3') and $\beta$ GUSr (5'-CCGAGTGAAGATCCCCTTTTTA-3') for the $\beta$-glucoronidase gene $\beta$ GUS, and TBPf (5'-GCCCGAAACGCCG AATAT-3') and TBPr (5'-CCGTGGTTCGTGGCTCTCT-3') for the TATA-box binding protein gene TBP. Relative mRNA levels were calculated by normalization of target gene mRNA level to the mean of the expression level of two endogenous reference genes ( $\beta$ GUS and TBP). For the calculation of delogarithmized relative expression values the mean relative expression of every target gene (the mean $\Delta \mathrm{C}_{\mathrm{T}}$-value) across all samples was set equal to one. All individual values were normalized to this value.

\section{Results}

Immunohistochemistry. The results of the immunohistochemical analysis are summarized in Table I. Three of the five neuroblastoma cell lines grown in vitro reacted with antihuman NCAM mAb (LAN-1, LAN-5, SH-SY5Y), whereas Kelly and SK-N-SH cells were negative for NCAM (Fig. 1a). The NCAM-positive cells also showed a moderate staining 
a

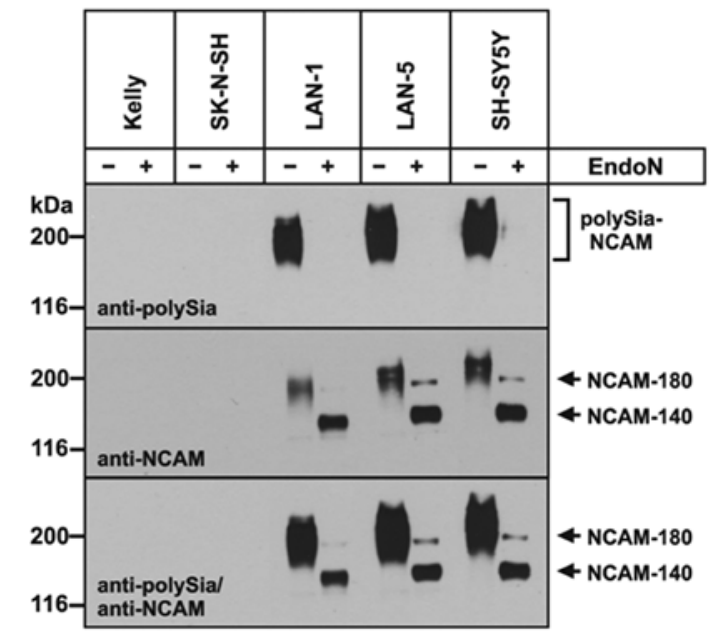

$\mathrm{b}$

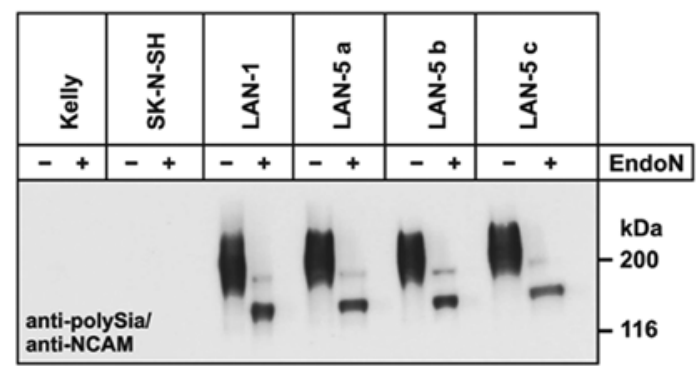

Figure 2. Western blot analysis of NCAM and polySia levels in human neuroblastoma cells grown in vitro (a) and in SCID mice (b). Lysates were separated before (-) and after (+) endoN treatment by 7\% SDS-PAGE. (a) Western blot analysis of cell grown in vitro. Blots were stained with antipolySia mAb 735 (upper panel), anti-NCAM mAb 123C3 (middle panel) or a combination of both antibodies (lower panel). In the case of Kelly and SK-N-SH 10 -fold the amount of total protein $(50 \mu \mathrm{g})$ was loaded per lane compared to LAN-1, LAN-5 and SH-SY5Y. Bands corresponding to the major NCAM isoforms NCAM-140 and NCAM-180 are indicated by arrows. Kelly and SK-N-SH were negative for NCAM and polySia, whereas LAN-1, LAN-5 and SH-SY5Y showed strong expression of polySia-NCAM. EndoN treatment revealed the expression of NCAM-140 and NCAM-180. (b) Western blot analysis of cells grown in SCID mice. Equal amounts of total protein were separated by SDS-PAGE and Western blot analysis was performed with anti-polySia mAb 735 and anti-NCAM mAb 123C3. For LAN-5, three independent samples were analyzed. Kelly and SK-N-SH were negative for both polySia and NCAM, whereas LAN-1 and LAN-5 showed strong expression of polySia-NCAM of the isoforms NCAM-140 and NCAM-180.

with anti-polySia antibody and the NCAM negative neuroblastoma cells were also negative for polySia (Fig. 1b). SK-N-SH and Kelly primary tumours grown in SCID mice were also negative for NCAM and polySia. LAN-5 tumours reacted moderately with anti-NCAM antibody as well as with antipolySia antibody (Fig. 1c and d). LAN-1 tumours showed inter-individual variations with weak or moderate NCAM and moderate polySia expression. SH-SY5Y tumours could not be examined because these cells did not adequately grow in SCID mice.

Western blot analyses. The NCAM isoform pattern and polysialylation status of all neuroblastoma cell lines used in this study were analysed by Western blotting using $\mathrm{mAb} 735$ specific for $\alpha 2,8$-linked polySia with $\geq 8$ residues $(25,27)$ and
Table I. Results of immunohistochemistry on human neuroblastoma cells grown in vitro and in SCID mice.

\begin{tabular}{lccccc}
\hline & \multicolumn{2}{c}{ Cells grown in vitro } & & \multicolumn{2}{c}{ Primary tumours } \\
\cline { 2 - 3 } \cline { 6 - 6 } Cell line & NCAM & PolySia & & NCAM & PolySia \\
\hline Kelly & - & - & & - \\
SK-N-SH & - & - & - & - \\
LAN-1 & ++ & ++ & + or ++ & ++ \\
LAN-5 & ++ to +++ & ++ & ++ & ++ \\
SH-SY5Y & ++ & ++ & no data & no data \\
\hline
\end{tabular}

-, negative; +, weak staining; ++, moderate staining; +++, strong staining.

$\mathrm{mAb} 123 \mathrm{C} 3$ recognizing all isoforms of human NCAM (24). Antibodies were either applied separately (Fig. 4a, upper and middle panel) or combined in order to allow enhanced detection of polySia-NCAM (Fig. 2a, lower panel and Fig. 2b). In LAN-1, LAN-5 and SH-SY5Y staining with mAb 735 revealed a broad high molecular weight signal typical for polysialylated NCAM (Fig. 2a, upper panel). In the case of Kelly and SK-N-SH 10-fold higher amounts of protein were applied compared to LAN-1, LAN-5 and SH-SY5Y. However, even under these conditions no polySia was detectable in Kelly and SK-N-SH. To analyze the NCAM isoform pattern, a parallel blot was stained with anti-NCAM antibody (Fig. 2a, middle panel). Clear NCAM signals were detected in the highly polySia-positive cell lines LAN-1, LAN-5 and SH-SY5Y and removal of polySia by endoN treatment resulted in the appearance of two focused bands with an apparent molecular mass of 140 and $180 \mathrm{kDa}$, indicating the expression of the two transmembrane isoforms NCAM-180 and -140. By contrast, Kelly and SK-N-SH were completely devoid of both polySia and NCAM and even after combined application of antipolySia and anti-NCAM mAb no signals were observed (Fig. 2a, lower panel).

Western blot analysis of cells xenografted in SCID mice (Fig. 2b) revealed that LAN-1 and LAN-5 were still positive for polySia-NCAM and displayed the same isoform pattern as the corresponding cells grown in vitro. For Kelly and SK-N-SH neither polySia nor NCAM could be detected. As mentioned above, SH-SY5Y tumours could not be examined due to the fact that SH-SY5Y cells did not adequately grow in SCID mice.

NCAM and polysialyltransferase transcript levels. Parallel to the Western blot analyses, all neuroblastoma cell lines were characterized by quantitative real-time RT-PCR using genespecific primers for NCAM and the two polysialyltransferases ST8SiaII and ST8SiaIV. Primers specific for NCAM transcripts were designed for amplification of all possible splice variants. Consistent with the protein expression profiles, high levels of NCAM mRNA were detected in LAN-1, LAN-5 and SH-SY5Y, whereas very low or no mRNA expression was observed in Kelly and SK-N-SH (Fig. 3a). Highest expression levels of ST8SiaII mRNA were detected in the highly polySia- 
a

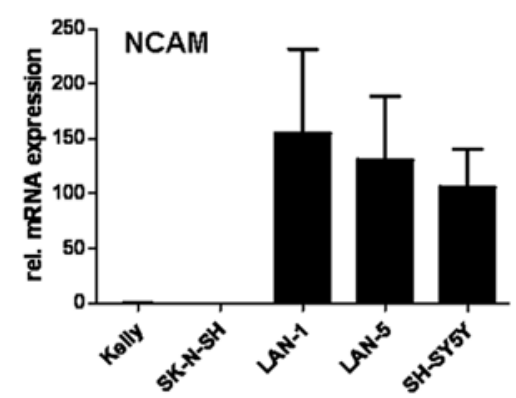

b

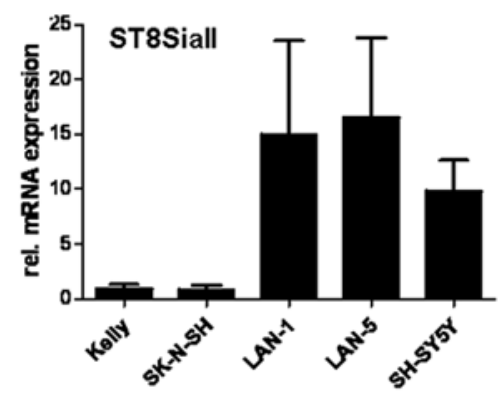

$\mathrm{C}$

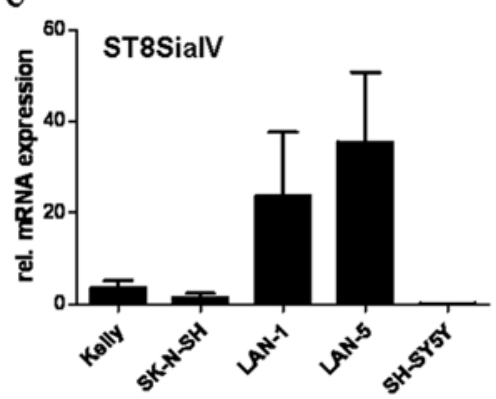

d

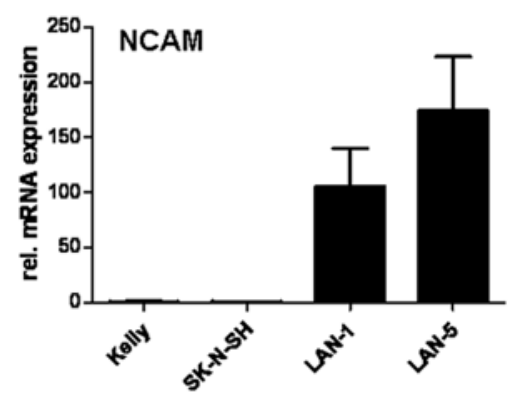

$\mathrm{e}$

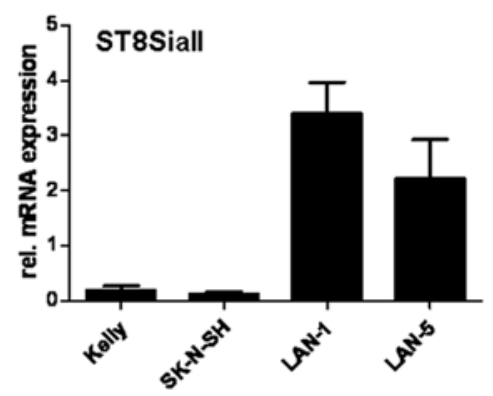

f

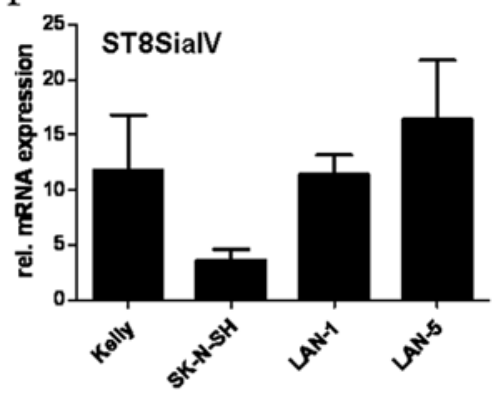

Figure 3. Quantitative RT-PCR analysis of transcript levels of NCAM (a and d), ST8SiaII (b and e) and ST8SiaIV (c and f) in neuroblastoma cells grown in vitro (a-c) and in SCID mice (d-f). All measurements were performed at least two times independently. Shown are the mean expression levels of all measurements $\pm \mathrm{SD}$.

positive cell lines LAN-1, LAN-5 and SH-SY5Y (Fig. 3b). LAN-1 and LAN-5 displayed also the highest ST8SiaIV transcript levels, whereas SH-SY5Y cells were almost devoid of ST8SiaIV mRNA (Fig. 3c). Compared to cells grown in vitro, the corresponding xenograft tumours showed similar relative mRNA expression levels for NCAM (Fig. 3d), ST8SiaII (Fig. 3e) and ST8SiaIV (Fig. 3f). Major variations were limited to ST8SiaIV transcript level in Kelly. Compared to LAN-1, the ST8SiaIV level in Kelly was 5-fold lower when cells were grown in vitro, whereas similar expression levels were observed when cells were grown in SCID mice (compare Fig. 3c and f).

Correlation of polySia-NCAM expression with tumour takerates and metastatic pattern. All NCAM-negative cell lines showed high take-rates after engraftment in SCID mice, whereas the highly polySia-NCAM-positive lines LAN-1, LAN-5 and SH-SY5Y were characterized by reduced take rates $(<40 \%)$ (Table II). In the case of SH-SY5Y, which showed the highest polySia level, no tumour formation was observed. Lung metastases were found for both polySia-NCAM negative (Kelly and SK-N-SH) and positive (LAN-1 and LAN-5) tumours. However, disseminated micrometastases in the alveolar septae were exclusively observed in animals with polySia-positive xenografts (Table II), indicating that if polySia-positive tumours grew in SCID mice, their pattern of metastases differed from NCAM-negative tumours which developed multicellular metastases located in the pulmonary artery and their tributaries (Fig. 4).

\section{Discussion}

In this study expression of polySia and NCAM in human neuroblastoma cells grown in vitro and in a SCID mouse model was investigated. In vitro, cells of three out of the five tested neuroblastoma cell lines were clearly positive for NCAM, namely LAN-1, LAN-5 and SH-SY5Y. Likewise, primary tumours that were obtained by inoculation of LAN-1 and LAN-5 in SCID mice reacted with anti-NCAM and antipolySia antibodies.

In a lung carcinoma xenograft model, sublines positive or negative for polySia showed different tumour growth. While 

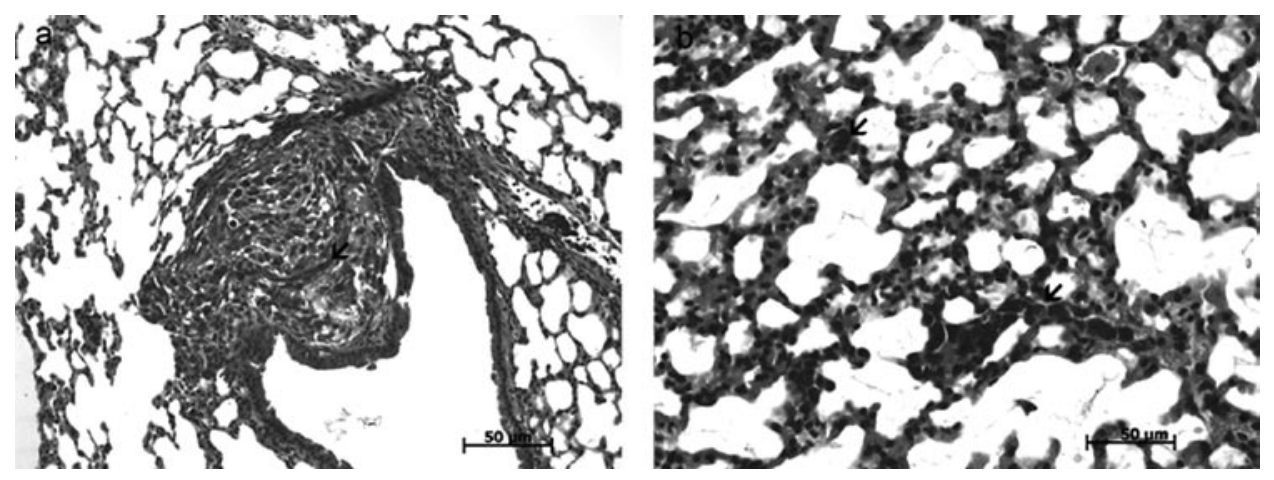

Figure 4. Pattern of metastasis formation amongst human neuroblastoma cells engrafted in SCID mice (HE staining). (a) PolySia-negative SK-N-SH tumours produced solid lung metastasis within a branch of the pulmonary artery. The arrow marks the intact smooth vascular vessel wall. (b) PolySia-positive LAN-1 tumours developed micrometastases (arrow), which are diffusely distributed in the alveolar septae of the lung.

Table II. PolySia-NCAM expression, take rates and pattern of metastasis of different human neuroblastoma cell lines xenografted in SCID mice.

\begin{tabular}{|c|c|c|c|c|c|}
\hline Cell line & NCAM $^{\mathrm{a}}$ & PolySia $^{a}$ & $\begin{array}{c}\text { Tumour take-rate } \\
(\%)\end{array}$ & Lung metastases ${ }^{\mathrm{b}}$ & $\begin{array}{c}\text { Disseminated } \\
\text { micrometastases }^{\mathrm{b}}\end{array}$ \\
\hline Kelly & Negative & Negative & $8 / 10(80)$ & + & - \\
\hline SK-N-SH & Negative & Negative & 9/9 (100) & + & - \\
\hline LAN-1 & Positive & Positive & 4/11 (36) & + & + \\
\hline LAN-5 & Positive & Positive & $3 / 9 \quad(33)$ & + & + \\
\hline SH-SY5Yc & - & - & $0 / 10 \quad(0)$ & - & - \\
\hline
\end{tabular}

${ }^{a}$ Expression in primary tumours determined by Western blot analysis. ${ }^{b}$ Data are from Valentiner et al (23). ${ }^{\mathrm{c}}$ Cell line with high polySia and NCAM expression in vitro.

expression of polySia-NCAM reduced tumour formation in mice, the absence of polySia enhanced tumour growth (28). This inhibitory effect of polySia expression on tumour formation is also confirmed by the engraftment of the neuroblastoma cells in our xenograft model. Neuroblastoma cell lines, which were strongly positive for NCAM and polySia (LAN-1, LAN-5 and SH-SY5Y) had lower tumour take-rates than the polySia-NCAM-negative cell lines (Kelly and SK-N-SH) (23).

NCAM isoform expression in neuroblastomas has been associated with differentiation status. A recent study assumed that benign tumours express the adhesive NCAM-120 isoform, while malignant neuroblastomas rather express NCAM-140 involved in neurite outgrowth and NCAM-180 involved in cell motility (4). In accordance with these findings, the NCAM-positive neuroblastoma cells analyzed in the present study showed highest expression of NCAM-140 followed by NCAM-180, two isoforms that are mainly expressed in less differentiated or malignant cell types $(4,6,7)$. NCAM-120, which is predominantly found in normal and differentiated tissue (6), could not be detected in our samples.

NCAM can be post-translationally modified by the addition of polysialic acid (polySia), a linear $\alpha$ 2,8-linked sialic acid polymer, which is covalently attached to the NCAM molecule $(29,30)$. In our study, NCAM-positive neuroblastoma cells were consistently positive for polySia and the polySia-NCAM positive cells LAN-1, LAN-5 and SH-SY5Y were characterized by the highest expression levels of ST8SiaII mRNA (Fig. 3b).
LAN-1 and LAN-5 displayed also the highest ST8SiaIV mRNA levels (Fig. 3c). ST8SiaII and ST8SiaIV are the key enzymes in the biosynthesis of polySia (17). Polysialylation of NCAM is crucial for NCAM-mediated regulation of tumour growth (31). In clinical studies high polySia serum levels were associated with poor prognosis and polySia and ST8SiaII were suggested as molecular prognostic markers $(15,16,21,32)$. PolySia is thought to abrogate homophilic binding properties of NCAM and to reduce cell adhesion important in cell migration and invasion. Thus, downregulation of NCAM as well as polysialylation of NCAM facilitate the migration of the cells from the primary tumour $(11,12)$.

Here all neuroblastoma cell lines that were engrafted in SCID mice developed lung metastases independent of polySiaNCAM expression. However, the pattern of metastatic disease was different for the individual cell lines. Thus, LAN-1 and LAN-5 cells developed numerous micrometastases in the alveolar septae, whereas Kelly and SK-N-SH cells produced multicellular metastases mostly located in the pulmonary artery and their tributaries (23). Comparing polySia-NCAM and ST8SiaII expression of the associated primary tumours, LAN-1 and LAN-5 tumours showed a high polySia-NCAM and ST8SiaII expression. This result supports the current model that expression of polySia-NCAM reduces the adhesiveness of tumour cells, contributes to cellular dissemination and may promote metastasis $(21,33)$. Cells from neuroblastomas, which are positive for polySia-NCAM disseminate and 
emigrate as single cells in the parenchyma of the lung, whereas cells of tumours without or with weak polySia-NCAM expression leave the primary tumour and adhere as cell clusters to the vascular vessel but do not transmigrate or do so very slowly, respectively.

Polysialic acid is the key regulator of NCAM-mediated interactions (34). By masking the underlying protein scaffold, polySia prevents not only homophilic NCAM-NCAM binding but also heterophilic interactions involved in signal transduction processes. In neuroblastoma cells, enzymatic removal of polySia from NCAM leads to the activation of extracellular signal-regulated kinase (ERK), resulting in reduced cell proliferation and induction of neuronal differentiation (31). In the present study, polySia was found exclusively on the 140and $180-\mathrm{kDa}$ isoforms of NCAM. Expression of these isoforms in their polysialylated state seems to reduce take-rates after subcutaneous engraftment of neuroblastoma cells in SCID mice and to have an influence on pattern of metastasis formation. In line with previous reports showing that neuroblastoma cell lines are either negative for NCAM or express the polysialylated form of NCAM (31), none of the cell lines used in our study was characterized by the expression of 'naked' polySia-free NCAM. Therefore, it was not possible to compare the malignant potential of polySia-NCAM-positive xenografts with those lacking polySia but expressing 'naked' NCAM. However, the developed xenograft model forms the basis for further studies addressing this question by manipulating the expression level of the polysialyltransferases.

\section{Acknowledgements}

We would like to thank Maike Hartmann, Britta Hasemeier, Christine Knies, Maike Märker and Renate Gehrcke for excellent technical support. This work was supported by grants of the Dr Mildred Scheel Stiftung für Krebsforschung.

\section{References}

1. Maris JM, Hogarty MD, Bagatell R and Cohn SL: Neuroblastoma Lancet 369: 2106-2120, 2007.

2. Berthold F, Hero B, Kremens B, Handgretinger R, Henze G, Schilling FH, Schrappe M, Simon T and Spix C: Long-term results and risk profiles of patients in five consecutive trials (1979-1997) with stage 4 neuroblastoma over 1 year of age. Cancer Lett 197: 11-17, 2003.

3. Jensen $\mathrm{M}$ and Berthold F: Targeting the neural cell adhesion molecule in cancer. Cancer Lett 258: 9-21, 2007.

4. Winter C, Pawel B, Seiser E, Zhao H, Raabe E, Wang Q, Judkins AR, Attiyeh E and Maris JM: Neural cell adhesion molecule (NCAM) isoform expression is associated with neuroblastoma differentiation status. Pediatr Blood Cancer 51: $10-16,2008$.

5. Walsh FS and Doherty P: Glycosylphosphatidylinositol anchored recognition molecules that function in axonal fasciculation, growth and guidance in the nervous system. Cell Biol Int Rep 15: 1151-1166, 1991.

6. Cavallaro U and Christofori G: Multitasking in tumor progression: signaling functions of cell adhesion molecules. Ann NY Acad Sci 1014: 58-66, 2004.

7. Crnic I, Strittmatter K, Cavallaro U, Kopfstein L, Jussila L, Alitalo K and Christofori G: Loss of neural cell adhesion molecule induces tumor metastasis by up-regulating lymphangiogenesis. Cancer Res 64: 8630-8638, 2004.

8. Rao Y, Wu XF, Gariepy J, Rutishauser U and Siu CH: Identification of a peptide sequence involved in homophilic binding in the neural cell adhesion molecule NCAM. J Cell Biol 118: 937-949, 1992.
9. Rutishauser U: Polysialic acid in the plasticity of the developing and adult vertebrate nervous system. Nat Rev Neurosci 9: 26-35, 2008.

10. Hildebrandt H, Muhlenhoff M and Gerardy-Schahn R: Polysialylation of NCAM. Neurochem Res 10.1007/s1106411008-19724-11067, 2008.

11. Blaheta RA, Hundemer M, Mayer G, Vogel JU, Kornhuber B, Cinatl J, Markus BH, Driever PH and Cinatl J Jr: Expression level of neural cell adhesion molecule (NCAM) inversely correlates with the ability of neuroblastoma cells to adhere to endothelium in vitro. Cell Commun Adhes 9: 131-147, 2002.

12. Blaheta RA, Daher FH, Michaelis M, Hasenberg C, Weich EM, Jonas D, Kotchetkov R, Doerr HW and Cinatl J Jr: Chemoresistance induces enhanced adhesion and transendothelial penetration of neuroblastoma cells by down-regulating NCAM surface expression. BMC Cancer 6: 294, 2006.

13. Suzuki M, Nakayama J, Suzuki A, Angata K, Chen S, Sakai K, Hagihara K, Yamaguchi Y and Fukuda M: Polysialic acid facilitates tumor invasion by glioma cells. Glycobiology 15: 887-894, 2005.

14. Tanaka F, Otake Y, Nakagawa T, Kawano Y, Miyahara R, Li M, Yanagihara K, Nakayama J, Fujimoto I, Ikenaka K and Wada H: Expression of polysialic acid and STX, a human polysialyltransferase, is correlated with tumor progression in non-small cell lung cancer. Cancer Res 60: 3072-3080, 2000.

15. Glüer S, Zense M, Radtke E and von Schweinitz D: Polysialylated neural cell adhesion molecule in childhood ganglioneuroma and neuroblastoma of different histological grade and clinical stage. Langenbecks Arch Surg 383: 340-344, 1998.

16. Glüer S, Schelp C, Madry N, von Schweinitz D, Eckhardt M and Gerardy-Schahn R: Serum polysialylated neural cell adhesion molecule in childhood neuroblastoma. Br J Cancer 78: 106-110, 1998.

17. Mühlenhoff M, Eckhardt M and Gerardy-Schahn R: Polysialic acid: three-dimensional structure, biosynthesis and function. Curr Opin Struct Biol 8: 558-564, 1998.

18. Ong E, Nakayama J, Angata K, Reyes L, Katsuyama T, Arai Y and Fukuda M: Developmental regulation of polysialic acid synthesis in mouse directed by two polysialyltransferases, PST and STX. Glycobiology 8: 415-424, 1998.

19. Hildebrandt H, Becker C, Murau M, Gerardy-Schahn R and Rahmann H: Heterogeneous expression of the polysialyltransferases ST8Sia II and ST8Sia IV during postnatal rat brain development. J Neurochem 71: 2339-2348, 1998.

20. Oltmann-Norden I, Galuska SP, Hildebrandt H, Geyer R, Gerardy-Schahn R, Geyer H and Muhlenhoff M: Impact of the polysialyltransferases ST8SiaII and ST8SiaIV on polysialic acid synthesis during postnatal mouse brain development. J Biol Chem 283: 1463-1471, 2008.

21. Cheung IY, Vickers A and Cheung NK: Sialyltransferase STX (ST8SiaII): a novel molecular marker of metastatic neuroblastoma. Int J Cancer 119: 152-156, 2006.

22. Schumacher U and Mitchell BS: Use of clinically relevant human-SCID-mouse models in metastasis research. Trends Biotechnol 15: 239-241, 1997.

23. Valentiner U, Valentiner FU and Schumacher U: Expression of CD44 is associated with a metastatic pattern of human neuroblastoma cells in a SCID mouse xenograft model. Tumour Biol 29: 152-160, 2008

24. Moolenaar CE, Muller EJ, Schol DJ, Figdor CG, Bock E, BitterSuermann D and Michalides RJ: Expression of neural cell adhesion molecule-related sialoglycoprotein in small cell lung cancer and neuroblastoma cell lines H69 and CHP-212. Cancer Res 50: 1102-1106, 1990.

25. Frosch M, Gorgen I, Boulnois GJ, Timmis KN and BitterSuermann D: NZB mouse system for production of monoclonal antibodies to weak bacterial antigens: isolation of an $\mathrm{IgG}$ antibody to the polysaccharide capsules of Escherichia coli K1 and group B meningococci. Proc Natl Acad Sci USA 82: 1194-1198, 1985.

26. Stummeyer K, Dickmanns A, Muhlenhoff M, Gerardy-Schahn R and Ficner R: Crystal structure of the polysialic acid-degrading endosialidase of bacteriophage K1F. Nat Struct Mol Biol 12: 90-96, 2005.

27. Hayrinen J, Haseley S, Talaga P, Muhlenhoff M, Finne J and Vliegenthart JF: High affinity binding of long-chain polysialic acid to antibody, and modulation by divalent cations and polyamines. Mol Immunol 39: 399-411, 2002.

28. Jimbo T, Nakayama J, Akahane K and Fukuda M: Effect of polysialic acid on the tumor xenografts implanted into nude mice. Int J Cancer 94: 192-199, 2001. 
29. Korja M, Jokilammi A, Salmi TT, Kalimo H, Pelliniemi TT, Isola J, Rantala I, Haapasalo H and Finne J: Absence of polysialylated NCAM is an unfavorable prognostic phenotype for advanced stage neuroblastoma. BMC Cancer 9: 57, 2009.

30. Mühlenhoff M, Oltmann-Norden I, Weinhold B, Hildebrandt $\mathrm{H}$ and Gerardy-Schahn R: Brain development needs sugar: the role of polysialic acid in controlling NCAM functions. Biol Chem 390: 567-574, 2009.

31. Seidenfaden R, Krauter A, Schertzinger F, Gerardy-Schahn R and Hildebrandt H: Polysialic acid directs tumor cell growth by controlling heterophilic neural cell adhesion molecule interactions. Mol Cell Biol 23: 5908-5918, 2003.
32. Glüer S, Schelp C, von Schweinitz D and Gerardy-Schahn R: Polysialylated neural cell adhesion molecule in childhood rhabdomyosarcoma. Pediatr Res 43: 145-147, 1998.

33. Fukuda M: Possible roles of tumor-associated carbohydrate antigens. Cancer Res 56: 2237-2244, 1996.

34. Weinhold B, Seidenfaden R, Rockle I, Muhlenhoff M, Schertzinger F, Conzelmann S, Marth JD, Gerardy-Schahn R and Hildebrandt $\mathrm{H}$ : Genetic ablation of polysialic acid causes severe neurodevelopmental defects rescued by deletion of the neural cell adhesion molecule. J Biol Chem 280: 42971-42977, 2005. 